\title{
Symbols and units
}

$\begin{array}{lll}\alpha & 1 / \mathrm{m} & \text { Absorption coefficient } \\ \alpha & \circ & \text { Angle } \\ A & \mathrm{~m}^{2} & \text { Area, cross section } \\ A & 1 & \text { Absorptance } \\ \beta & \circ & \text { Angle } \\ d & \mathrm{~m} & \text { Diameter, distance, layer thickness } \\ E & \mathrm{~W} / \mathrm{m}^{2} & \text { Irradiance } \\ E & \mathrm{~J} & \text { (Electric) energy } \\ E_{\lambda} & \mathrm{W} / \mathrm{m}^{3} & \text { Spectral irradiance } \\ \mathrm{FF} & 1 & \text { Fill factor } \\ H & \mathrm{MJ} / \mathrm{m}^{2}\left(\mathrm{kWh} / \mathrm{m}^{2}\right) & \text { Irradiation }(1 \mathrm{kWh}=3.6 \mathrm{MJ}) \\ I & \mathrm{~A} & \text { Current } \\ K_{\tau \alpha} & 1 & \text { Incidence angle modifier } \\ L & \mathrm{~m} & \text { Edge length } \\ \mathrm{AM} & 1 & \text { Air mass } \\ n & 1 & \text { Refractive index or counting index } \\ P & \mathrm{~W} & \text { Power } \\ \mathrm{PR} & 1 & \text { Performance ratio (of PV module or PV power plant) } \\ \mathrm{QE} & 1 & \text { Quantum efficiency } \\ R & 1 & \text { Reflectance } \\ r & \Omega & \text { Sheet resistivity } \\ \rho & \Omega \cdot \mathrm{m} & \text { Electrical (volume) resistivity } \\ T & 1 & \text { Transmittance } \\ T & { }^{\circ} \mathrm{C}, \mathrm{K} & \text { Temperature in degree Celsius or in Kelvin as indicated } \\ V & \mathrm{~V} & \text { Voltage } \\ V & \mathrm{~m} / \mathrm{s} & \text { Velocity } \\ w & \mathrm{~m} & \text { Width }\end{array}$

\section{Subscripts}

$\begin{array}{ll}\text { a } & \text { activation } \\ \text { AC } & \text { Alternating current } \\ \text { beam } & \text { Beam or direct (irradiance, irradiation) } \\ \text { Bulk } & \text { Referring to the material volume, without interfaces } \\ \text { cell } & \text { Referring to the cell } \\ \text { DC } & \text { Direct current } \\ \text { diff } & \text { Diffuse (irradiance, irradiation) } \\ \text { e } & \text { Electron } \\ \text { eff } & \text { Effective } \\ \text { enc } & \text { Encapsulant } \\ \text { ext } & \text { External } \\ \mathrm{f} & \text { fitted } \\ \text { glob } & \text { Global (irradiance, irradiation) } \\ \text { h } & \text { Hydrolisis } \\ \text { int } & \text { Internal }\end{array}$




$\begin{array}{ll}\text { m } & \text { measured } \\ \text { max } & \text { maximum } \\ \text { MPP } & \text { PV module } \\ \text { OC } & \text { Maximum power point } \\ \text { ox } & \text { Open circuit } \\ \text { P } & \text { Production } \\ \text { PD } & \text { Photodegradation } \\ \text { plant } & \text { PV power plant } \\ \text { POA } & \text { Plane of the array } \\ \text { red } & \text { reduced } \\ \text { ref } & \text { reference } \\ \text { res } & \text { residual } \\ \text { SC } & \text { Short circuit or solar constant } \\ \text { STC } & \text { Standard testing conditions } \\ \text { T } & \text { Thermal } \\ \text { Tm } & \text { Thermomechanical } \\ \text { TIR } & \text { Total internal reflectance } \\ \text { U } & \text { Usage }\end{array}$

\section{Abbreviations}

$\begin{array}{ll}\text { AR } & \text { Antireflective } \\ \text { AES } & \text { Auger electron spectroscopy } \\ \text { AFM } & \text { Atomic force microscopy } \\ \text { AM } & \text { Air mass } \\ \text { AS } & \text { Antisoiling } \\ \text { ASTM } & \text { American Society for Testing and Materials } \\ \text { B2B } & \text { Business to business } \\ \text { B2C } & \text { Business to customer } \\ \text { BOM } & \text { Bill of materials } \\ \text { BOS } & \text { Balance of System } \\ \text { BST } & \text { Black standard temperature } \\ \text { CB } & \text { Certification body } \\ \text { CdTe } & \text { Cadmium telluride } \\ \text { CED } & \text { Cumulated energy demand } \\ \text { CIE } & \text { Commission Internationale de l'Ecleirage } \\ \text { CIS } & \text { Copper indium selenide } \\ \text { C-Si } & \text { Crystalline silicon } \\ \text { CPV } & \text { Concentrating PV } \\ \text { CTE } & \text { Coefficient of thermal expansion } \\ \text { CTM } & \text { Cell to module } \\ \text { DH } & \text { Damp heat } \\ \text { DIN } & \text { Deutsches Institut für Normung - German Standardization Organization } \\ \text { DKE } & \text { Deutsche Kommission Elektrotechnik - German Electrotechnical Commission } \\ \text { DMA } & \text { Dynamic mechanical analysis } \\ \text { DoC } & \text { Degree of cross-linking } \\ & \end{array}$




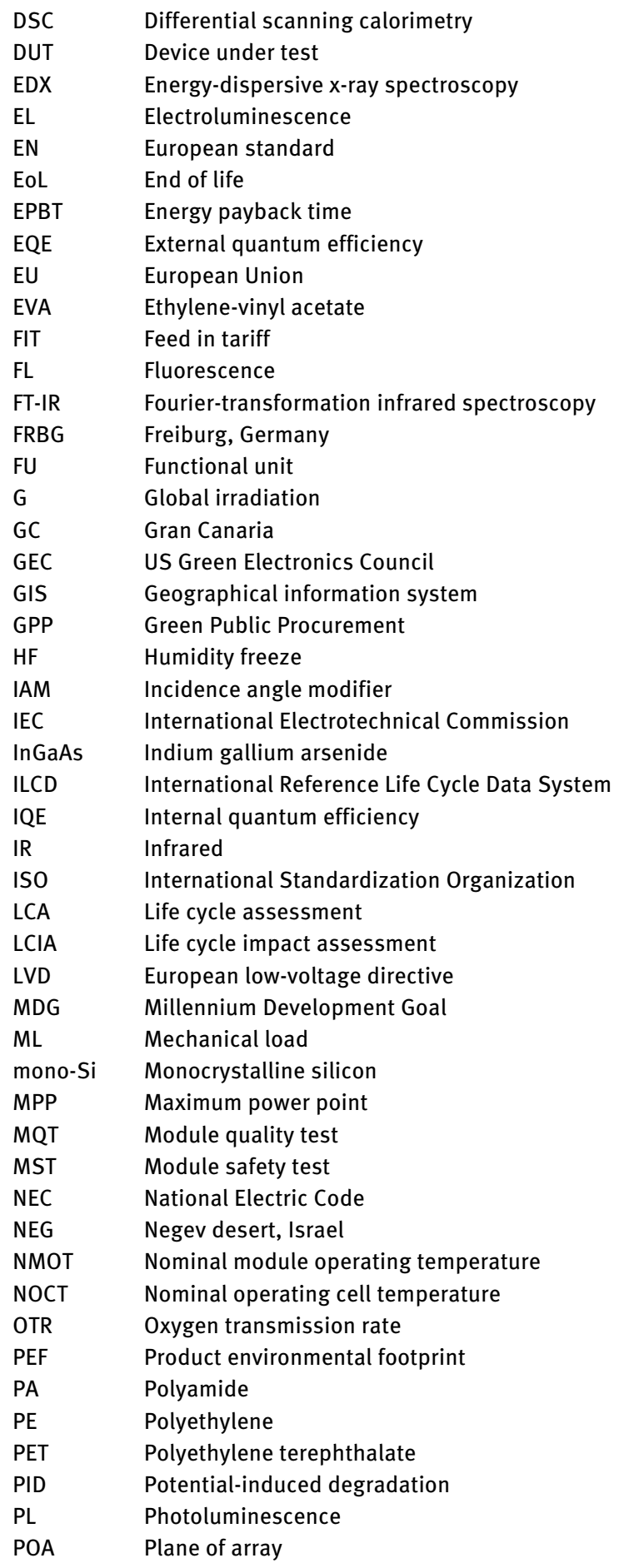


POE Polyolefin elastomers

poly-Si Poly- or multicrystalline silicon

PP Polypropylene

PV Photovoltaic(s)

PVB Polyvinyl butyral

PVDF Polyvinylidene fluoride

PVF Polyvinyl fluoride

QA Quality assurance

RH Relative humidity

SAM Scanning acoustic microscopy

SD Standard deviation

SDG Sustainable developments goal

STC Standard testing conditions

TC Temperature cycling

TCO Total cost of ownership

TF Thin film

TMF Thermomechanical fatigue

TOW Time of wetness

TPSE Thermoplastic silicone elastomer

TPT Backsheet laminate with layers Tedlar-PET-Tedlar

UFS Environmental Research Station on the mountain Zugspitze, Germany

UN United Nations

UV Ultraviolet

vis Visual

WEEE Waste electrical and electronic equipment directive

WST White standard temperature

WVD Water vapor diffusion

WVTR Water vapor transmission rate

YI Yellowness Index 